\title{
Conformance Testing and Analysis of Synchrophasor Communication Message Structures and Formats for Wide Area Measurement Systems in Smart Grids
}

\author{
Adeyemi Charles Adewole, Raynitchka Tzoneva \\ Centre for Substation Automation and Energy Management Systems (CSAEMS), Cape Peninsula University of \\ Technology, South Africa
}

\section{Article Info}

Article history:

Received Apr 4, 2017

Revised May 11, 2017

Accepted May 18, 2017

\section{Keyword:}

Communication network IEEE C37.118

Phasor Measurement Unit

Smart grids

Synchrophasors

ABSTRACT

The renewed quest for situational awareness in power systems has brought about the use of digital signal processing of power system measurements, and the transmission of such data to control centres via communication networks. At the control centres, power system stability algorithms are executed to provide monitoring, protection, and control in order to prevent blackouts. This can be achieved by upgrading the existing Supervisory Control and Data Acquisition (SCADA) systems through the deployment of newly proposed power system synchrophasor-based applications for Wide Area Monitoring, Protection, and Control (WAMPAC). However, this can only be done when there is a complete understanding of the methods and technologies associated with the communication network, message structure, and formats required. This paper presents an analysis of the IEEE C37.118 synchrophasor message framework, message formats, and data communication of synchrophasor measurements from Phasor Measurement Units (PMUs) for WAMPAC schemes in smart grids. A newly designed labscale testbed is implemented and used in the practical experimentation relating to this paper. Synchrophasor measurements from the PMUs are captured using a network protocol analyzer software-Wireshark, and the compliance of the synchrophasor message structures and formats captured was compared to the specifications defined in the IEEE C37.118 synchrophasor standard.
\end{abstract}

Copyright @ 2017 Institute of Advanced Engineering and Science. All rights reserved.

\section{Corresponding Author:}

A.C. Adewole,

Centre for Substation Automation and Energy Management Systems,

Cape Peninsula University of Technology,

Symphony Way, Bellville 7535, Cape Town South Africa.

Email: charles.adewole@ieee.org

\section{INTRODUCTION}

Although, wide area blackouts are infrequent, their after-effects are usually devastating with far reaching consequences. Often times, they are the result of series of events occurring randomly in a local area until they cascade into a chain reaction that ultimately propagates into the wide area thereby causing blackouts or system-wide outages [1].

Following blackouts in parts of the USA and Canada, the North American SynchroPhasor Initiative (NASPI) was formed to improve power system reliability by using wide area measurement and control. Synchrophasor measurements are real-time phasor values of sinusoidal waveforms synchronized to a common time base such as the Global Positioning System (GPS).

Synchrophasors have found application in Wide Area Measurement Systems (WAMS) [2-3], power system stability monitoring/assessment [4-5], wide area protection and control [6-9], and model validation [10-11]. 
International standards such as the IEEE C37.118 synchrophasor standard have been drafted to amongst other functions, regulate data communication and information exchange of synchrophasor measurements in power systems. However, manufacturers (vendors) of PMU devices do not issue specification sheets on the message structure and formats implemented in their devices. This information is important in the design and implementation of synchrophasor-based wide area monitoring systems. Therefore, it is necessary to carry out comparisons and compliance of vendor equipment to the IEEE C37.118 standard in order to ensure the transfer of valid critical synchrophasor information.

This paper presents a detailed practical experimentation and analysis of the synchrophasor message structure and formats captured from three PMUs from different vendors implemented in a newly developed lab-scale Wide Area Monitoring, Protection and Control testbed. Also, compliance with the IEEE Std C37.118 was investigated.

The rest of this paper is structured as follows: An overview of the structure and format of synchrophasor measurements is presented in Section 2. Section 3 describes the experimental setup and simulations carried out. Section 4 presents the verification of the synchrophasor message structure, analysis, and comparison of the measurements captured from the actual PMUs. Section 5 summarizes the contribution of this paper.

\section{SYNCHROPHASOR MEASUREMENT FORMAT}

\subsection{Wide Area Measurement Systems}

Most power systems around the world still utilise Supervisory Control and Data Acquisition (SCADA) system to provide data telemetry and telecontrol functions. SCADA systems are typically made up of sensors, meters, dataloggers, Remote Terminal Units (RTUs), Master Terminal Unit (MTU), Intelligent Electronic devices (IEDs), and Programmable Logic Controllers (PLCs). The RTUs collect field data from the sensors, meters, dataloggers, and IEDs, and transmits them to the MTU via a communication medium. The MTU displays the acquired data on the Human-Machine Interface (HMI), and also carries out remote control functions. Some of the communication protocols used in SCADA systems include Profibus, Modbus, DNP 3.0, IEC 60870-5-101, and IEC 60870-5-104. Figure 1 [12] shows a typical architecture of a SCADAbased system (Figure 1a) and a synchrophasor-based system (Figure 1b).

However, SCADA systems are limited by their slow data sampling rate, slow reporting rate, and the measurements obtained from various locations are not time synchronized. Wide Area Measurement Systems (WAMS) based on synchrophasor measurements provide faster sampling and reporting rates, and the measurements are synchronized to a common time reference. Also, synchrophasors provide direct measurements of the phasor angles in the power system.

Therefore, state estimation algorithms for estimating phasor angles are not required in synchrophasor-based WAMS. The major components of WAMS include PMUs/Phasor Measurement Control Units (PMCU), Phasor Data Concentrator (PDCs), Data Archiver (DA), satellite clock, and communication network infrastructure.

\subsection{Synchrophasor Standard}

The drafting of International standards for synchrophasors was as a result of the need to support the developments of PMUs and their ancillary equipment. Also important was the need to establish a common platform for vendors to use in the design and manufacture of synchrophasor devices.

The first synchrophasor standard published was the IEEE Std 1344-1995 [13]. The standard was intended to introduce synchrophasors and define the basic concepts of measurements and data transfer. Sources of precise time synchronization, time-tag for data samples, and message formats were also contained in this standard [13].

The IEEE Std 1344-1995 was reaffirmed in 2001 and replaced by the IEEE Std C37.118-2005 [14] in 2005. The IEEE Std C37.118-2005 provides additional clarification for phasor and synchronized phasor definitions. Also, the standard introduced the concepts of Total Vector Error (TVE) to verify the compliance of phasor estimates with theoretical equivalents for the same time instants. Furthermore, compliance tests and data reporting rates that are submultiples of the nominal system frequency were defined. The message formats in the IEEE Std C37.118 was updated from the IEEE 1344-1995 standard to improve the exchange of information with other systems in higher hierarchies, such as a PDC. This includes the addition of the sync, frame size, and station identification fields to the data, configuration, header, and command message types. The data frame also includes an additional field for analogue data [14].

The latest version of the synchrophasor standard was released in 2011 to replace the 2005 standard. It was split into IEEE Std C37.118.1 for measurements and C37.118.2 for data transfer [15-16]. The C37.118.1-2011 standard is divided into 5 clauses and 6 annexes [15]. While the C37.118.2-2011 standard 
has 6 clauses and 6 annexes [16]. The IEEE Std C37.118.1-2011 has clauses that cover the scope and the needs of the standard, definition of the measurements, measurement requirements, quantification of the measurement, testing of the measurements, and definition of the accuracy limits. The 6 annexes provide information on the bibliography, time tagging, the algorithms used to confirm the performance requirements, time synchronization, Total Vector Error (TVE), and measurement of generator internal voltages and power angles.

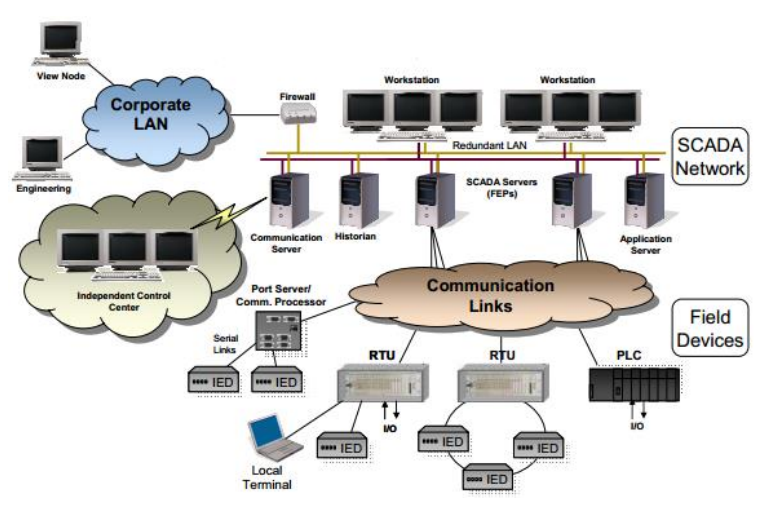

(a)

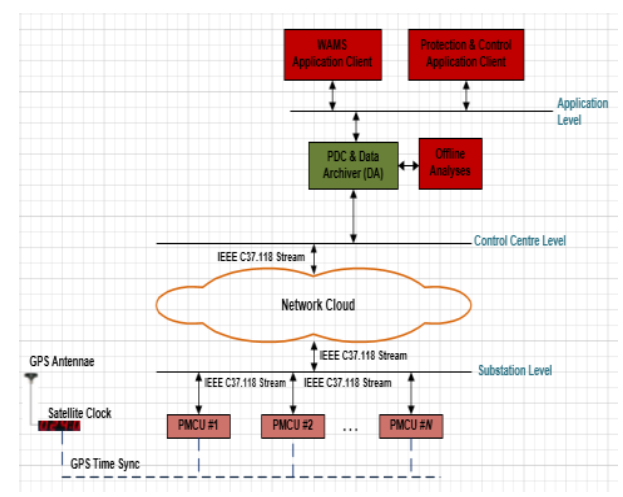

(b)

Figure 1. Wide Area Measurement Architecture Using (a) SCADA-Based Measurements; (b) SynchrophasorBased Measurement

Similarly, the IEEE Std C37.118.2-2011 covers the scope of the standard, background information on synchrophasor measurements, synchrophasor measurement systems, communication protocol, and message formats. The annexes contain information on the bibliography, Cyclic Redundancy Check (CRC), communication bandwidth, message format, message mapping, and communication methods using Internet Protocol (IP).

The modifications included in the IEEE Std C37.118.1 include new definitions for the phasors, frequency, and Rate of Change of Frequency (ROCOF), Frequency Error (FE), ROCOF Error (RFE), performance requirement for dynamic conditions, and the introduction of two performance classes-the $M$ class and the $\mathrm{P}$ class. The $\mathrm{P}$ class is for applications that require fast response e.g. protection applications. While the $\mathrm{M}$ class is for applications that require higher accuracy e.g. metering [15]. Total Vector Error (TVE) requirements and the compliance tests are expanded in this standard by adding new temperature and dynamic performance tests.

The IEEE Std C37.118.2 defines synchrophasor data transfer requirements, clarifications and modification regarding data transfer, etc. The standard presents the structure of the message, defines the words used in the message and specifies the numbers of bytes per word. Also, the standard defines the time quality, organization of the data, configuration, header, and command frames [16].

\subsection{Data communications and synchrophasor message format}

The IEEE Std C37.118 does not specify the transport protocol to use in the communication of synchrophasor measurements as long as the measurement and performance criteria are satisfied. The communication of PMUs to PDCs can be done using Ethernet-based and serial communication protocols. The Ethernet-based transport layer protocols commonly used are the Transport Communication Protocol (TCP) and the User Datagram Protocol (UDP). The communication medium can be leased lines, microwave, Synchronous Optical Network, (SONET), etc.

Four types of messages are defined in the IEEE Std C37.118. These include data, configuration, header, and command messages [16-17]. The data, configuration, and header messages are transmitted from a PMU/PDC (data source) to the PDC (data concentrator). While the command messages are sent from the PDC (data concentrator) and received by the PMU/PDC (data source). It should be noted that the PDC normally serves as a C37.118 client. However, it can act as a C37.118 server when it outputs the concentrated time-aligned measurements hitherto received from the PMUs, to other PDCs in a higher hierarchy.

The data frame contains the synchrophasor measurements computed by the PMU or PDC. It includes an identification header, message length, message source ID, status information, and the data itself. 
The data includes the phasors, frequency, ROCOF, analogue, and digital data types.

The configuration frame is a machine-readable dataset containing information relating to a synchrophasor data stream. Three types of configuration frames are defined in the IEEE Std C37.118. These are Config1 (CFG-1), Config2 (CFG-2), and Config3 (CFG-3). Config1 configuration frame is used to give the capability of the PMU/PDC (data source), and it provides all the data that the PMU/PDC is capable of reporting. CFG-1 has a fixed length frame with 19 fields excluding the data rate and the CRC. It is denoted by bits 4-6 (010) of the frame synchronization word (SYNC), with fields 8-19 defining the capability of the PMU(s). Config2 configuration frame is used to give the measurements currently being reported in the data frame by the PMU/PDC (data source). CFG-2 is a fixed length frame with 19 fields excluding the data rate and the CRC. It is denoted by bits 4-6 (011) of the frame synchronization word (SYNC), with fields 8-19 defining the measurements from the PMU(s). Config3 is an optional configuration frame, and is used to give the measurements currently being reported in the data frame by the PMU/PDC (data source). CFG-3 is an extensible variable length frame with 27 fields excluding the data rate and the CRC. It is denoted by bits 4-6 (101) of the frame synchronization word (SYNC), with fields 9-27 defining the measurements from the PMU(s).

The header frame is a human-readable frame in ASCII format sent from the data source to the data concentrator. It is user configured and contains the information relating to the PMU, type of algorithm, data sources, type of signal processing, and scaling. The command frame is sent from the data concentrator to the data source to start/stop the transmission of data, or to request for configuration data prior to data transmission.

The tests and analyses carried out in this paper are performed in the following order: i). Design and implementation of a lab-scale hardware-in-the-loop wide area monitoring, protection and control testbed; ii). Configuration of the communication network and software required for communication network analysis; iii). Packet sniffing of the synchrophasor measurements off the communication network; and v). Analyses and verification of conformance of the message framework and formats obtained from PMUs from various vendors with the specifications defined in the IEEE C37.118 standard.

\section{LAB-SCALE EXPERIMENTAL SET-UP}

In A lab-scale hardware-in-the-loop experiment comprising of the Real-Time Digital Simulator ${ }^{\circledR}$ (RTDS), PMUs, PDCs, GPS satellite clock, industrial network switches, and network protocol analyzer is used for the investigations relating to this paper. Figure 2 depicts the lab-scale setup developed and implemented at the Centre for Substation and Energy Management Systems (CSAEMS) at the Cape Peninsula University of Technology.

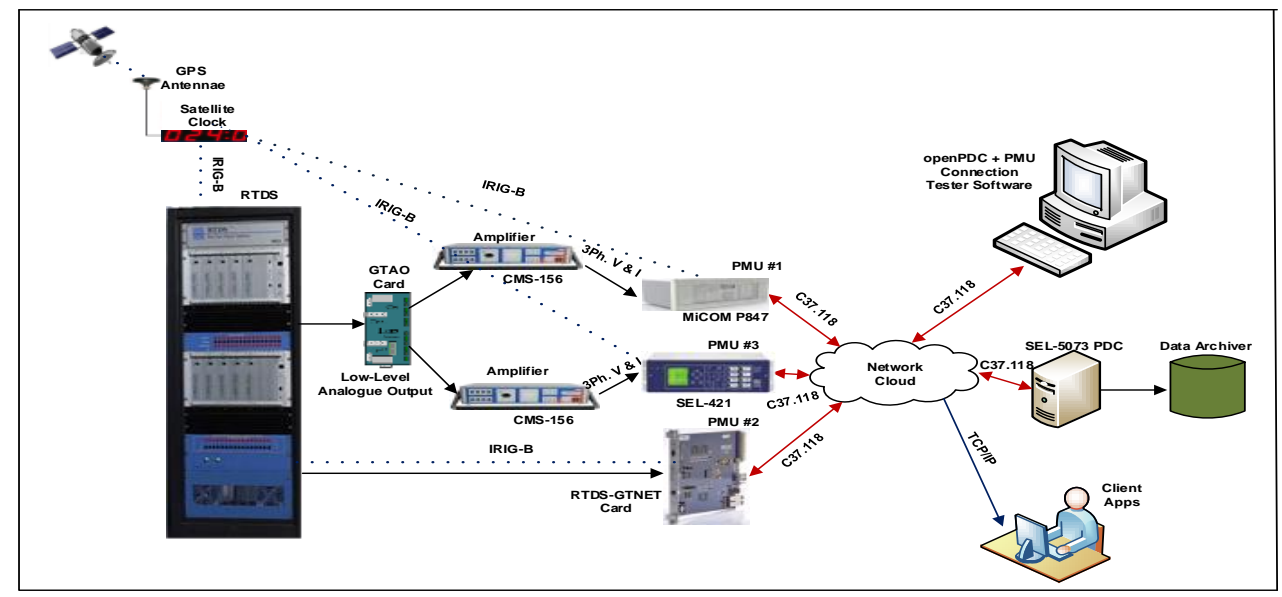

Figure 2. Structure of the Lab-Scale Testbed Implemented

The $\operatorname{RTDS}^{\circledR}$ is used in simulating a typical electric power system in real-time. Measurement variables from the simulation are exported to external devices (Intelligent Electronic Devices (IEDs) or PMUs) via an amplifier (Omicron CMS-156 amplifier) which then serves as the analogue inputs to the IEDs/PMUs. Three types of PMUs are used. They are MiCOM P847 IED, RTDS-GTNET PMU, and the 
SEL-421 IED. These PMUs are denoted as PMUs 1-3 respectively. It should be noted that PMU-1 and PMU3 comply with the IEEE Std C37.118-2005, while PMU-2 complies with the IEEE Std C37.118-2011. The time synchronization source was demodulated IRIG-B time signal from a SEL-2407 satellite clock to all the PMUs. However, the MiCOM P847 also requires a 1 Pulse Per Second (1 PPS) signal in order to publish synchrophasor measurements.

The Phasor Data Concentrators (PDCs) used are the SEL-5073 PDC and the openPDC software respectively. The PDCs perform the task of concentration and time-alignment of the synchrophasor measurements coming from the PMUs. The study system used in publishing the synchrophasor measurements is modelled in RSCAD software, and the three PMUs are placed at a High Voltage (HV) transmission bus (bus-7) as shown in Figure 3 [1]. The PMUs are all connected to the same Instrument Transformers (sensors) at bus-7 in order to ensure that they receive the same primary quantities. The measurements of interest are published as IEEE C37.118 synchrophasor measurements by the PMUs to the communication network.

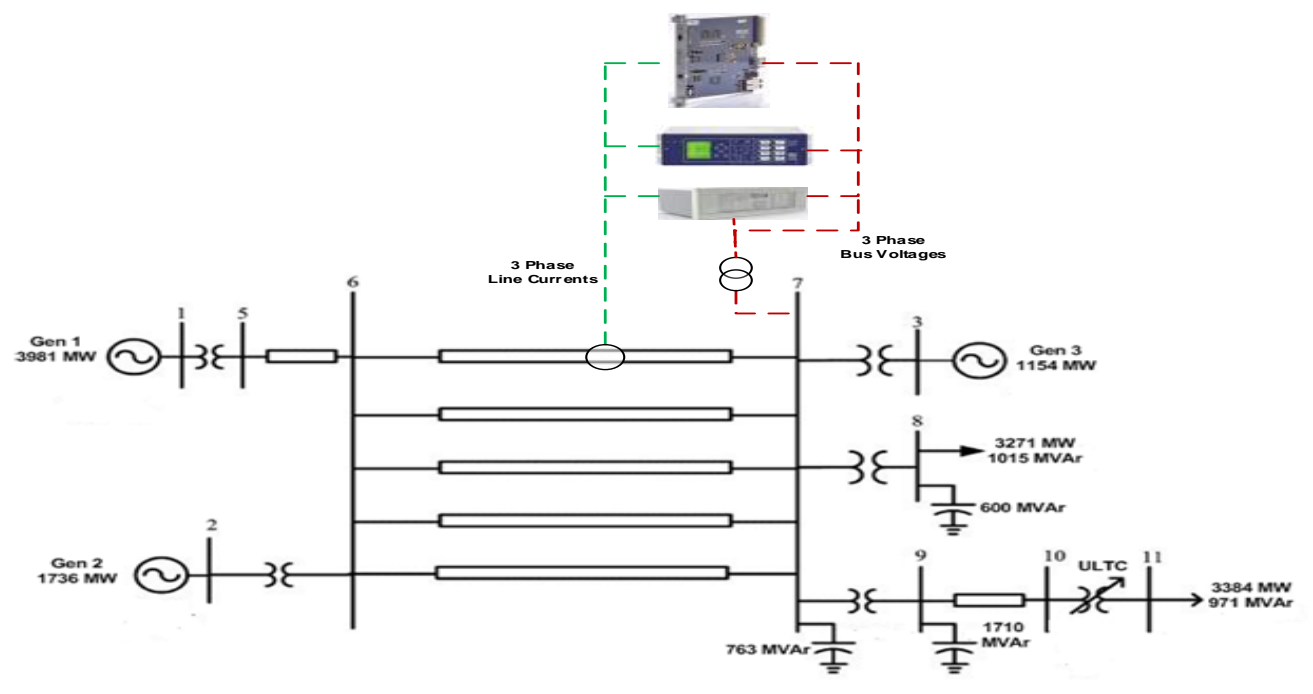

Figure 3. 10-Bus Multi-Machine Equivalent Power System

Table 1 gives some of the parameters used in the configuration of the PMUs and the synchrophasor stream published by each PMU. These consist of positive sequence voltage $\left(\mathrm{V}_{1}\right)$ and current $\left(\mathrm{I}_{1}\right)$, frequency, ROCOF, analogues, and a digital word. The communication protocol used by the PMUs is TCP. Figure 4 depicts the communication architecture for the investigations carried out.

Table 1. Configuration Settings for PMUs 1-3

\begin{tabular}{|c|c|c|c|}
\hline Configuration & PMU-1 & PMU-2 & PMU-3 \\
\hline Performance Class & - & Class P & 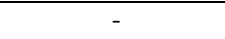 \\
\hline Configuration Frame Format & Config 2 & Config 2 & Config 2 \\
\hline Reporting Rate & $60 \mathrm{fps}$ & $60 \mathrm{fps}$ & $60 \mathrm{fps}$ \\
\hline Phasor Format & Real & Real & Real \\
\hline Phasor Output Format & Polar & Polar & Polar \\
\hline Phasor Output (Positive sequence) & $\mathrm{V}_{1} \& \mathrm{I}_{1}$ & $\mathrm{~V}_{1} \& \mathrm{I}_{1}$ & $\mathrm{~V}_{1} \& \mathrm{I}_{1}$ \\
\hline Analogue Format & Real & Real & Real \\
\hline Number of Phasors & 2 & 2 & 2 \\
\hline Number of Analogues & 0 & 0 & 8 \\
\hline Number of Digital & 1 & 0 & 1 \\
\hline $\begin{array}{c}\text { Size of Measurement in Data Frame } \\
(\text { Phasor + Freq + ROCOF + Analogues }+ \\
\text { Digital) bytes }\end{array}$ & $26(16+4+4+0+2)$ & $24(16+4+4+0+0)$ & $58(16+4+4+32+2)$ \\
\hline IP Address of PMU (Server) & 192.168.1.45 & 192.168.1.203 & 192.168.1.206 \\
\hline PMU Port Nr. & 4745 & 4710 & 4730 \\
\hline IP Address of PDC (Client) & 192.168.1.33 & 192.168.1.40 & 192.168 .1 .30 \\
\hline Configuration Frame Format & - & Class P & - \\
\hline
\end{tabular}




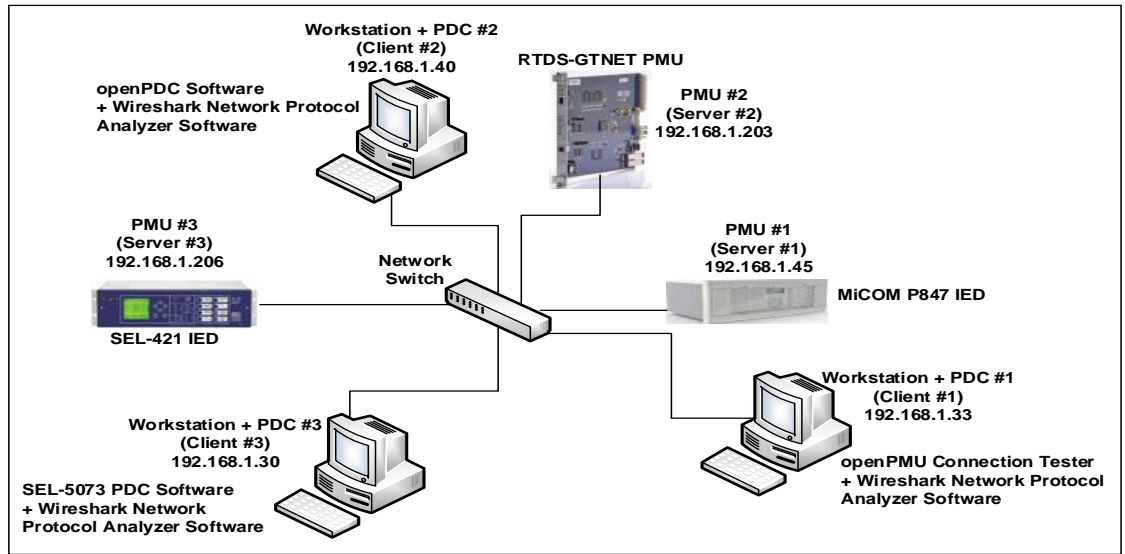

Figure 4. Communication Layout for the Investigations Carried Out

The configuration of the respective PMUs mainly comprise of the station name, hardware ID code, output port, phasor format, the phasors/analogues/digital quantities, reporting rate, and performance class $(\mathrm{P} / \mathrm{M})$. The PMUs are configured to stream positive sequence synchrophasor measurements of voltage and current calculated from their three-phase values.

The PMUs (MiCOM P847 IED, SEL-421, RTDS-GTNET PMU) streaming the synchrophasor measurements are referred to as Servers 1-3 respectively. In order to capture the synchrophasor message frames using Wireshark network protocol analyzer software, a client must subscribe to the data published by the PMUs. The SEL-5073 PDC, openPDC, and PMU Connection tester software are used for this purpose as illustrated in Figure 4, and are referred to as Clients 1-3 respectively. Wireshark network protocol analyzer running on each of the workstation connected to the communication network is then used in sniffing the relevant packets (synchrophasor frames) off the network.

\section{RESULTS AND DISCUSSION}

\subsection{Synchrophasor message structures}

The verification of the IEEE C37.118 synchrophasor message structure as given in the standard is carried out with respect to the three PMUs used in this investigation. Figure 5 shows the packets from PMU1 obtained using a network protocol analyser-Wireshark. Wireshark is used to snifsf the network, capture network packets in real-time, and display them in human-readable format. The figure has been partitioned into three panes (Panes A-C) for better analysis.

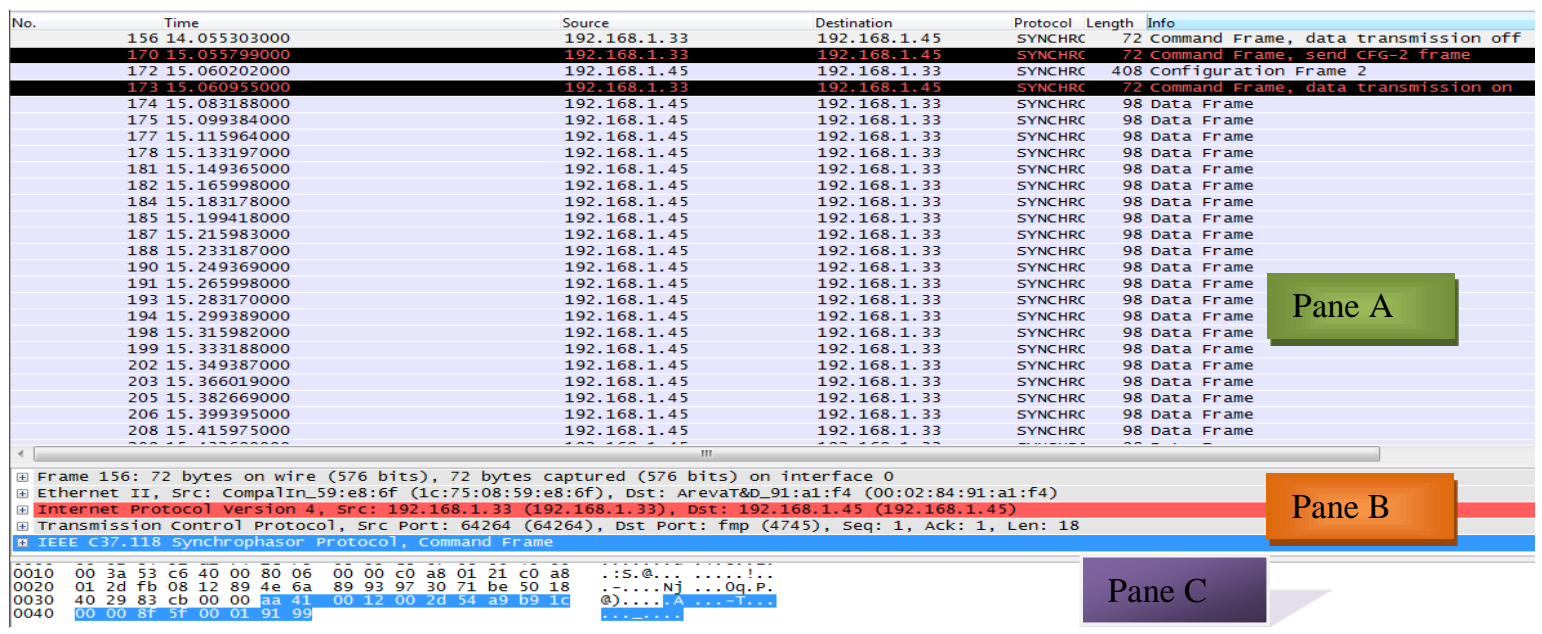

Figure 5. Synchrophasor Messages for PMU-1 and PDC-1 Captured Using a Network Protocol Analyzer-Wireshark 
Pane A has six columns showing the time of capture, the source, destination, type of protocol, frame length, and the message type. Column 1 gives the time of capture of the packet. The IP address of the source device is given in column 2. Column 3 presents the IP address of the destination device. The type of protocol was rightly given as SYNCHRC in column 4 signifying the synchrophasor protocol. The frame length gives the size of the information contained in the message type as shown in column 5.

As defined in the standard and as shown in Section 2.3 on synchrophasor message format, Column 6 of Pane A shows the information relating to the sequence of interaction between the PDC (data concentrator) and the PMU (data source). It can be seen that the PDC initiates a query (Command frame) requesting for the CFG-2 Configuration message from the PMU.

The PMU responds to the Command frame query by issuing its CFG-2 configuration, and shortly afterwards, commences the data transmission of synchrophasor measurements to the PDC. Pane B of the figure gives a layered classification of the selected packet in Pane A.

The hexadecimal values corresponding to the messages in the captured packet are given in Pane $\mathrm{C}$ of Figure 5. The above applies to the captured packets for PMUs 2-3 as shown in Figures 6-7 respectively.

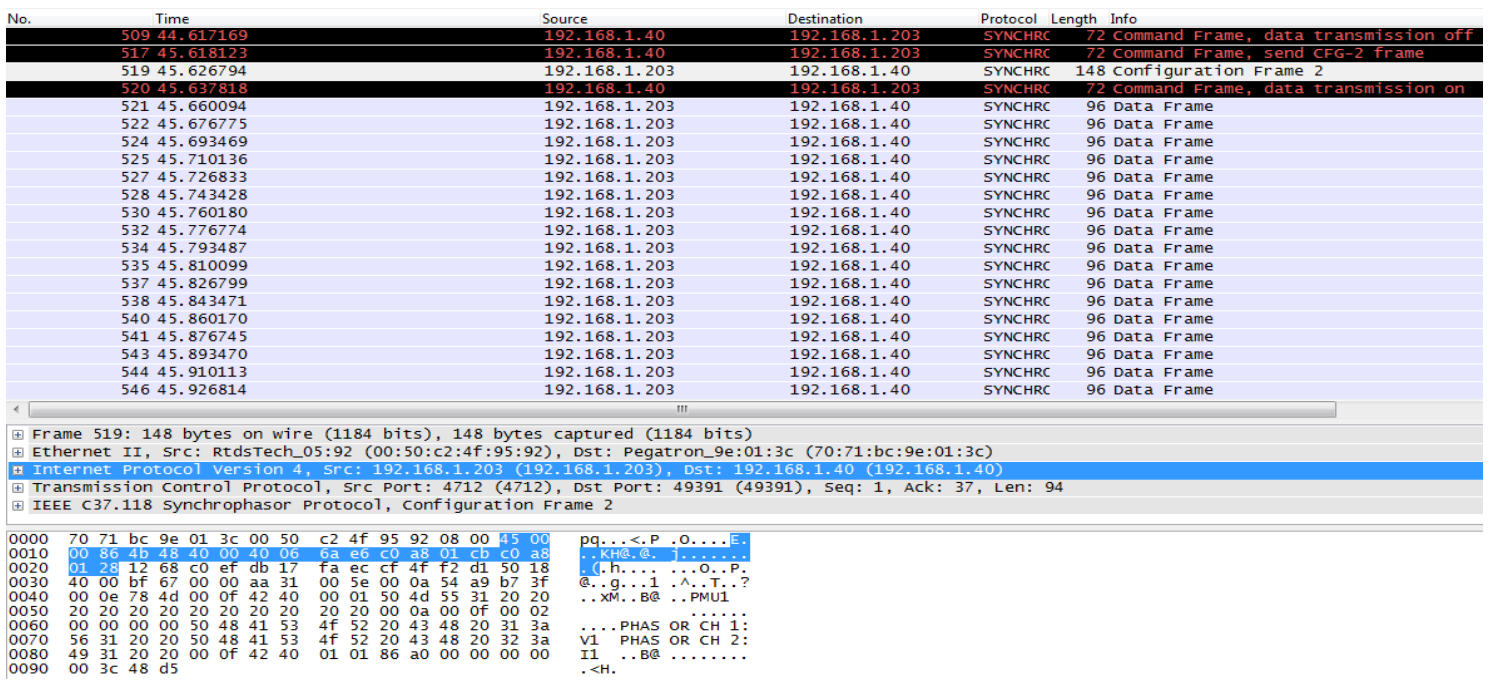

Figure 6. Synchrophasor Messages for PMU-2 and PDC-2 Captured Using A Network Protocol Analyzer-Wireshark

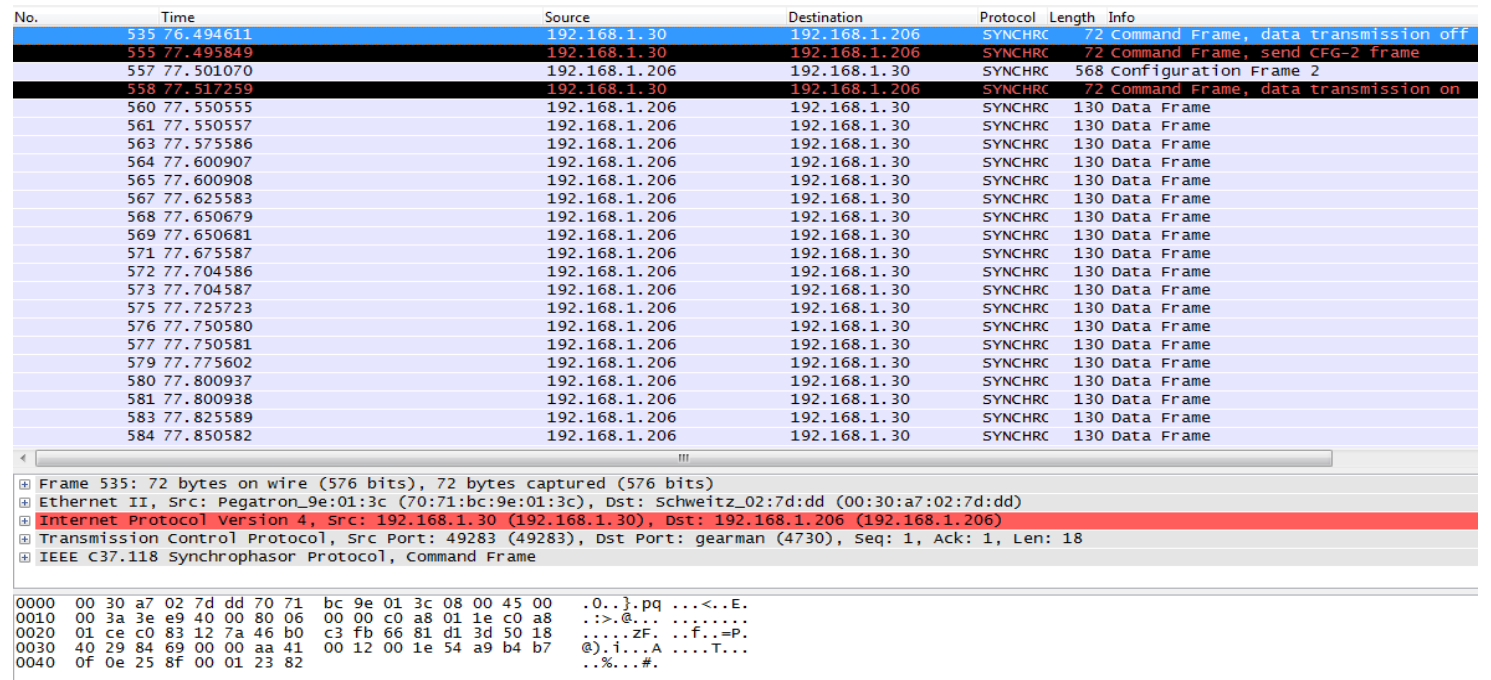

Figure 7. Synchrophasor Messages for PMU-3 and PDC-3 Captured Using A Network Protocol Analyzer-Wireshark 


\subsection{Synchrophasor message types}

The required fields of the command frame in the synchrophasor message are specified in Table 14 of the IEEE Std C37.118.2-2011. In order to verify compliance with the standard, a comparison between the fields in the IEEE Std C37.118.2-2011 and the Command frame from an actual PDC was carried out. The Command frame issued by each PDC to their respective PMU was captured using Wireshark network protocol analyzer as shown in Figures 8-9.

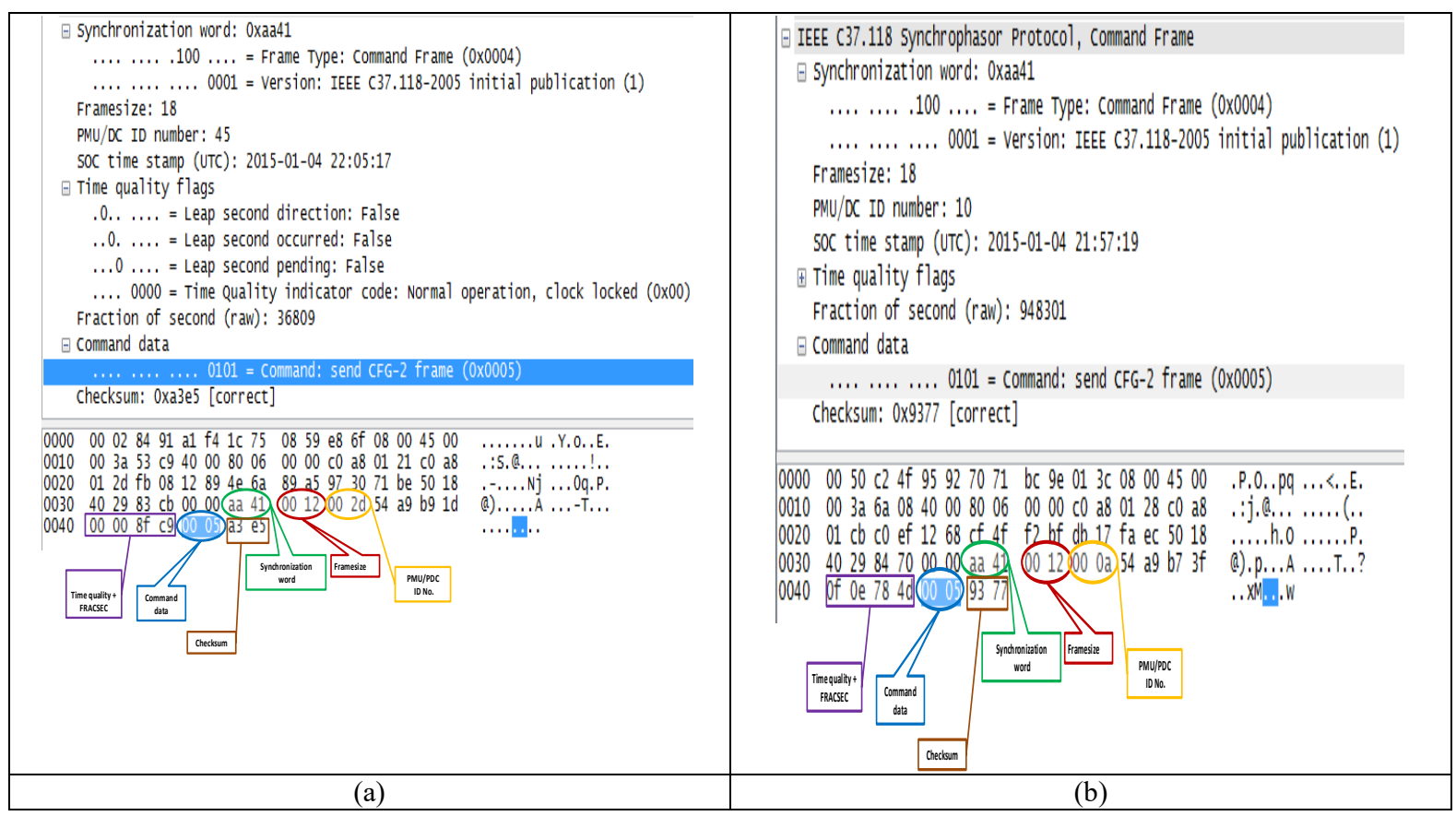

Figure 8. Wireshark Capture of Command Fram from (a) PDC-1 to PMU-1; (b) PDC-2 to PMU-2

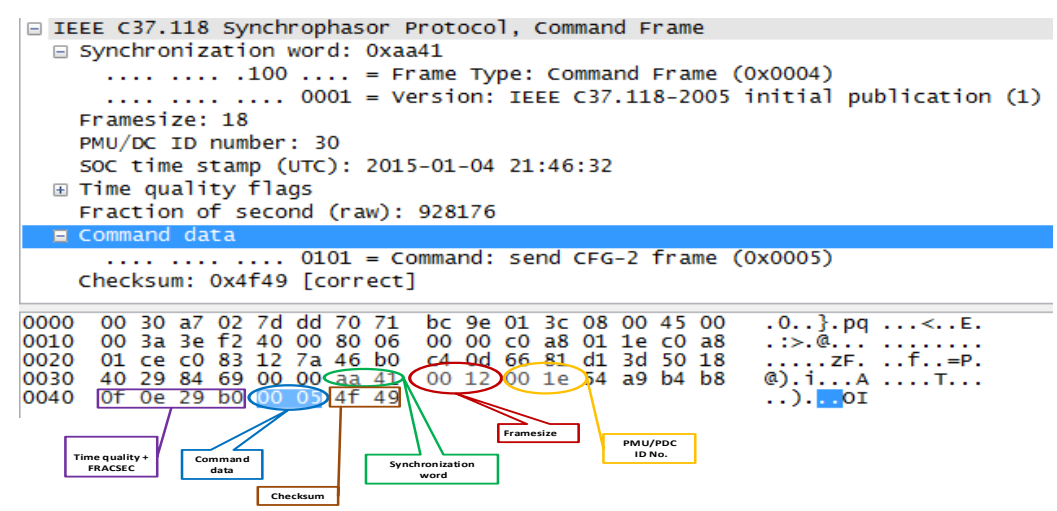

Figure 9. Wireshark Capture of Command Fram from PDC-3 to PMU-3

From Figures 8-9, the message fields contained in the command frame from the clients to the servers were shown to give information on the synchronization word, framesize, ID code, SOC, Fraction of Second (FRACSEC), command to the PMU, and the checksum. Similarly, the same message fields as specified in the IEEE Std C37.118 were obtained for the command frame from PDC-2 to PMU-2, and PDC-3 to PMU-3 respectively.

From the captured packets in Figures 8-9, compliance with the standard is verified in terms of the field contained in the message frame, and also the size of the field. Information relating to the frame synchronization word (sync), the message frame size, device ID code, SOC, FRACSEC, the command being sent, and the checksum can be seen in the Wireshark capture.

The configuration frame is a binary dataset containing the required parameters for processing the synchrophasor data stream. The fields in the configuration frame are specified in Table 8 of the IEEE Std 
C37.118.2-2011. It contains information relating to the SYNC word, framesize, ID code, SOC, FRACSEC. In addition to these, the configuration frame contains information relating to the data format (integer or floating point), phasor names, conversion factors for the phasors, configuration change count, reporting rate, and the checksum. The configuration frames published by PMUs- 2 and -3 are captured using Wireshark network protocol analyzer as shown in Figures 10-11. For lack of space, the Wireshark capture of the configuration frame for PMU-1 is not presented in this paper.

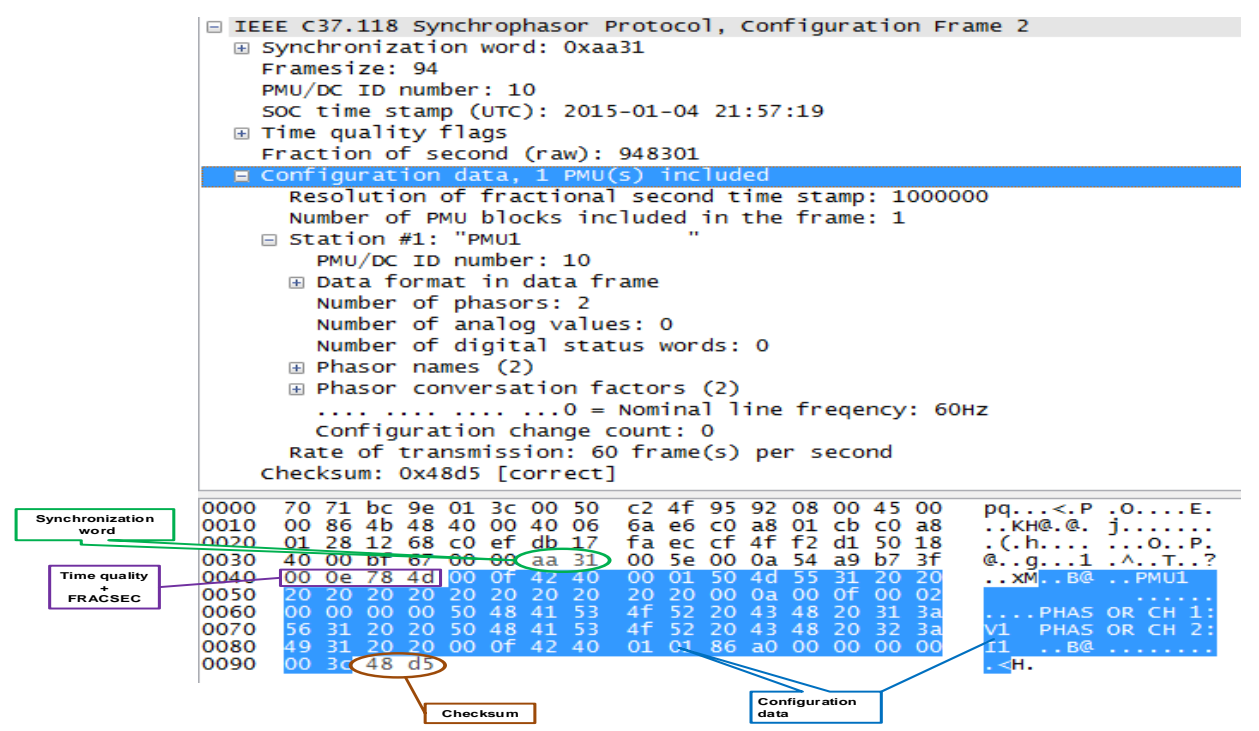

Figure 10. Configuration Frame Organization from the Captured Configuration Frame for PMU-2

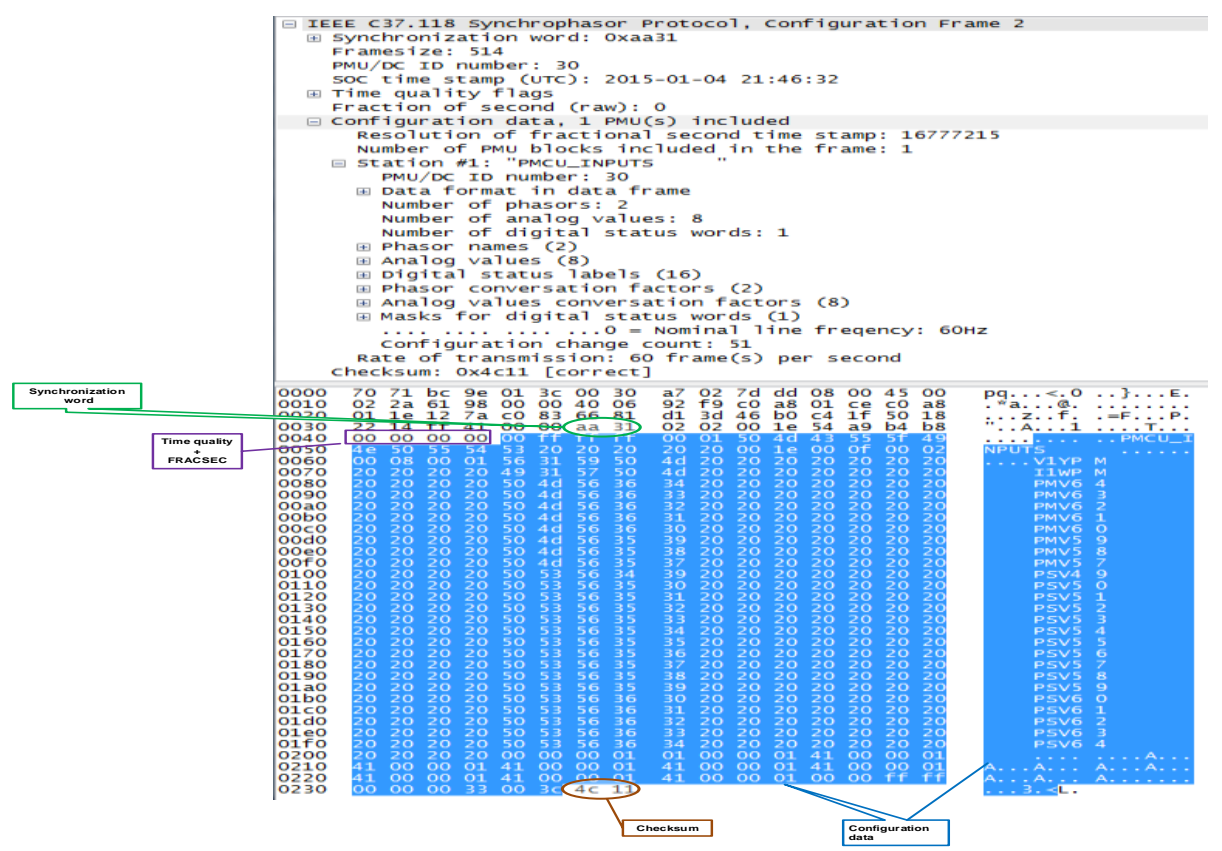

Figure 11. Configuration Frame Organization From the Captured Configuration Frame for PMU-3

The specified fields indicated in the configuration frame sent by the PMU/PDC are populated in the data frame. The fields consist of the synchronization word, framesize, ID code, SOC, FRACSEC, STAT, Phasors, Frequency, ROCOF, analogues, digitals, and checksum. The data frame packet captured using the network protocol analyzer is shown in Figure 12. 


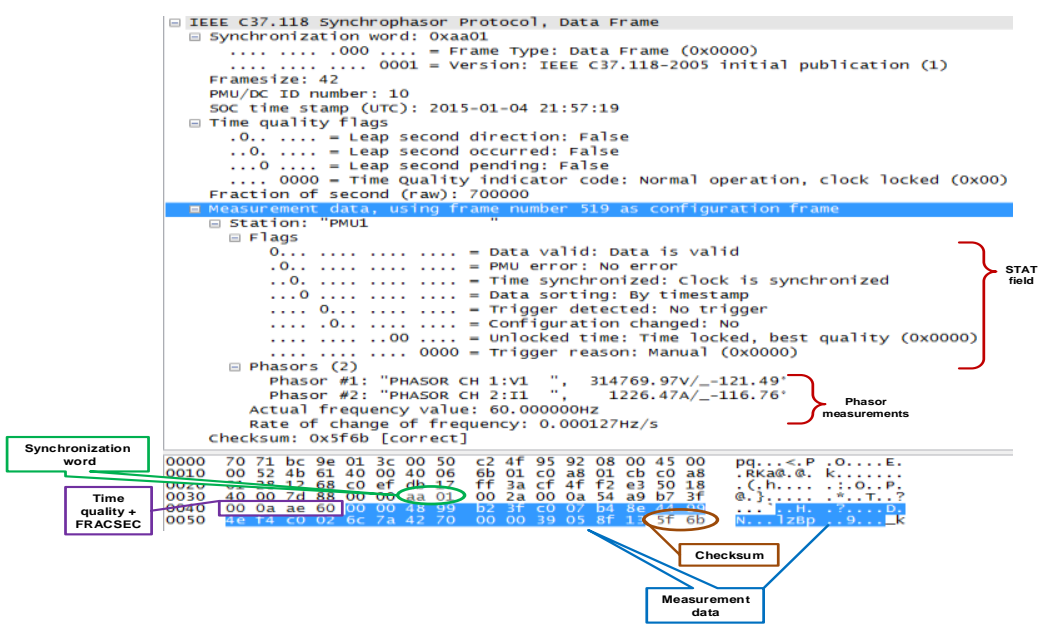

Figure 12. Data Frame Organization From The Captured Data Frame (PMU-2)

\subsection{Discussion}

For the Command frame, the fields in the captured network packet for the Command frames (send CFG-2 frame) shown in Figures 8-9 are further analysed. The fields and the word size were as specified in the IEEE Std C37.118.2-2011, except for the EXTFRAME field which is field 7 of Table 14 in the IEEE Std C37.118.2-2011 [16]. The EXTFRAME field was missing in the captured command frame for PMUs 1-3. This is because the EXTFRAME field is optional.

Also, it was observed that in field 5 of Table 14 of the IEEE Std C37.118.2-2011 [16], the size of the FRACSEC and time quality are bunched together as 4 bytes. Analysis done on the captured command frames from PMUs 1-3 shows the FRACSEC to be 3 bytes, while the time quality is 1 byte. The SYNC word is given as hex AA41 signifying the frame type and version number. The Send CFG-2 frame command is correctly given as 0000000000000101 as shown in Figures 8-9. Also, the time quality indicator should be noted as $0 \mathrm{x} 00$ (locked state) signifying a good time synchronization.

The checksum has a hex value of $0 \mathrm{x} 5 \mathrm{f} 6 \mathrm{~b}$ and is given as 'correct'. This implies that the data was received without any error. The command frame has a total of 18 bytes corresponding to the summation of the above-mentioned messages in compliance with the specification in Table 14 of the IEEE Std C37.118.2-2011 [16].

Another Command frame is the 'data transmission on' command. This is also issued by the PDC (data concentrator) to the PMU (data source). The only difference between this and the 'Send CFG-2' command is that the command word bits is 0000000000000010 , rather than 0000000000000101 which is the 'Send CFG-2' command.

Analysis of the fields obtained from the captured packet for the Configuration frame is given in Figures 10-11. From Figures 10-11, the SYNC word is given as hex AA31. This corresponds to CFG-2. All the PMUs report the FRACSEC and time quality separately, and their sizes are obtained in the captured packet as 3 bytes and 1 byte respectively.

Peculiar to the Configuration frame is the message content relating to the station name, data format in the data frame, number of phasors, number of analogue values, number of digital status words, phasor names, and phasor conversion factors. The packet size for PMU-2 for this particular configuration frame is 94 bytes, while PMU-3 has a framesize of 514 bytes because of its additional analogue measurements and digital word. The total size of the configuration frame is determined by the number of phasors, number of analogues, and the digital word being reported. The respective fields and their sizes were verified to be in accordance with the IEEE Std C37.118 [16] as shown in Figures 10-11.

The Data frame for PMU-2 is analysed using Figure 12. From the figure, the SYNC word is obtained as hex AA01. This corresponds to the version 1 IEEE Std C37.118-2005 frame. The FRACSEC and time quality in field 5 are separated and their sizes are obtained in the captured packet as 3 bytes and 1 byte respectively. Field 6 of Table 5 in the IEEE Std C37.118.2-2011 [16] is specified as 'STAT'. However, this is named as 'Flags' in PMUs 1-3. For the 'Flags' field, bits 15-14 have a value of 00 (good measurement data without errors), bit 13 equals 0 , thereby signifying PMU synchronization to a UTC traceable time source. Bit 12 is 0 for data sorting using time stamp rather than the order of arrival, bits 08-06 are 00, signifying a PMU time quality of 00 synchronized and locked with the best quality time, etc. The size of the data frame for this particular frame is 42 bytes. This includes the size of the SYNC word, framesize, ID code, SOC, time quality, FRACSEC, the measurement values of the phasors, frequency, ROCOF, analogues, and digitals statuses. 


\section{CONCLUSION}

This paper has presented a detailed interpretation of the message structure and contents of synchrophasor measurements from actual PMUs implemented in a hardware-in-the-loop testbed. The structures of synchrophasor messages were captured from three PMUs using a network protocol analyzer Wireshark, were analysed and compliance with the IEEE Std C37.118 was investigated.

The knowledge obtained from the analysis of the synchrophasor message structure and organization is required in communication network planning in smart grids in terms of bandwidth requirements, latency issues, communication protocol type, reliability requirements, and database archiving. Also, the contribution of this paper on the message structure of synchrophasors can serve as a foundation for designing missing data algorithms, security tools for network intrusion detection, and other applications. Furthermore, a working knowledge of the data organization is essential in the design of the algorithms utilizing the synchrophasor measurements as input for WAMPAC schemes in smart grids. Future work shall focus on the compliance of the measurements from various PMUs in terms of the TVE, FE, and RFE requirements under steady-state and dynamic conditions.

\section{ACKNOWLEDGEMENTS}

This research work is funded by the South African National research Foundation NRF THRIP Grant TP2011061100004 "CSAEMS development and growth".

\section{REFERENCES}

[1] P. Kundur, "Power System stability and control," McGraw-Hill, 1994.

[2] V. Salehi, et al., "Laboratory-Based Smart Power System, Part II: Control, Monitoring, and Protection," IEEE Transactions on Smart Grids, vol. 3, no. 3, pp.1405-1417, 2012. [Online]. Available: http://dx.doi.org/10.1109/TSG.2012.2194519

[3] L. Vanfretti, et al., "SmarTS Lab - A Laboratory for Developing Applications for WAMPAC Systems," IEEE PES General Meeting 2012, pp.1-8, July 2012. [Online]. Available: http://dx.doi.org/ 10.1109/PESGM.2012.6344839

[4] K. Sun, et al., "An Onlie Dynamic Security Assessment Scheme Using Phaasor Measurements and Decision Tree, IEEE Transactions on Power Systems, vol. 22, no. 4, pp.1935-1943, 2007. [Online]. Available: http://dx.doi.org/ 10.1109/TPWRS.2007.908476

[5] A. C. Adewole, R. Tzoneva, "Real-Time Deployment of a Novel Synchrophasor-Based Voltage Stability Assessment Algorithm," International Review of Electrical Engineering, vol. 9, no. 5, pp.1021-1033, 2014. [Online]. Available: http://dx.doi.org/10.15866/iree.v9i5.3051

[6] A. C. Adewole, R. Tzoneva, "Wide Area Voltage Stability Assessment Based on Generator-Derived Indices using Phasor Measurement Units," 2015 International Conference on the Industrial and Commercial Use of Energy (ICUE), pp. 291-298, 2015. [Online]. Available: http://dx.doi.org/10.1109/ICUE.2015.7280281

[7] C. W. Taylor, et al., "WACS-Wide-Area Stability and Voltage Control System: R\&D and Online demonstration," Proceedings of the IEEE, vol. 93, no. 5, pp.892-906, 2005. [Online]. Available: http://dx.doi.org/ 10.1109/JPROC.2005.846338

[8] V. Terzija, et al., "Wide-Area Monitoring, Protection, and Control of Future Electric Power Networks, Proceedings of the IEEE, vol. 99, no. 1, pp.80 -93, 2011. [Online]. Available: http://dx.doi.org/ 10.1109/JPROC.2010.2060450

[9] J. V. Espinoza, et al., "Wide Area Protection and Control Scheme," PACWorld Magazine, December 2013.

[10] J. Ma, et al., "Wide area measurements-based model validation and its application," IET Generation, Transmission, Distribution, vol. 2, no. 6, pp.906-916, 2008. [Online]. Available: http://dx.doi.org/ 10.1049/iet-gtd:20080177

[11] H. Bai, P. Zhang, V. Ajjarapu, "A novel parameter identification approach via hybrid learning for aggregate load modeling," IEEE Transactions on Power Systems, vol. 24, no. 3, pp.1145-1154, 2009. [Online]. Available: http://dx.doi.org/ 10.1109/TPWRS.2009.2022984

[12] The role of authenticated communications for electric power distribution, Pacific Northwest National Laboratory, (2006). [Available: http://www.science.upm.ro/ traian/web_curs/Scada/docum/SCADA_archit.pdf].

[13] IEEE Std 1344TM-1995, IEEE Standard for Synchrophasors for Power Systems.

[14] IEEE Std C37.118 http://dx.doi.org/10.1109/IEEESTD.2006.99376

[15] IEEE Standard C37.118.1 [Online]. Available: http://dx.doi.org/10.1109/IEEESTD.2011.6111219

[16] IEEE Standard C37.118.2 ${ }^{\text {TM}}-2011$, IEEE Standard for Synchrophasor Data Transfer for Power Systems, 2011. [Online]. Available: http://dx.doi.org/10.1109/IEEESTD.2011.6111222

[17] K. E. Martin, et al., "An Overview of the IEEE Standard C37.118.2-Synchrophasor Data Transfer for Power Systems," IEEE Transactions on Smart Grid, vol. 5, no. 4, pp.1980-1984, 2014. [Online]. Available: http://dx.doi.org/ 10.1109/TSG.2014.2302016 